\title{
Single and multiple solution algorithms to scan asteroid databases for identifications
}

\author{
Maria E. Sansaturio and O. Arratia \\ E.T.S. de Ingenieros Industriales, University of Valladolid, Paseo del Cauce s/n 47011 \\ Valladolid, Spain \\ email: genny@pisces.eis.uva.es
}

\begin{abstract}
The process of cataloguing the minor planet population of the Solar System has experienced a great advance in the last decades with the start-up of several surveys. The large volume of data generated by them has increased with time and given rise to huge databases of asteroids with uneven qualities.

In fact, a significant fraction of these objects have not been enough observed, thus leading to the computation of very poor quality orbits as to carry out useful predictions of the positions of such asteroids. As a result, some objects can get lost, which is particularly embarrassing for those with Earth crossing orbits.

When this situation persists for a long time, the aforementioned databases end up contaminated in the sense that they contain more than one discovery for the same physical object and some kind of action must be taken. The algorithms for asteroid identifications are thought precisely to mitigate this problem and their design will depend upon the quality of the available data for the objects to be identified.

In this paper we will distinguish two cases: when both objects have a nominal orbit and when one of them lacks it. In addition, when the available data poorly constrain the solution, other orbits in the neighbourhood of the nominal one are also compatible with the observations. Using these alternative orbits allows us to find many identifications that otherwise would be missed. Finally, we will show the efficiency of all these algorithms when applied to the datasets distributed by the Minor Planet Center.
\end{abstract}

Keywords. Asteroids, catalogs; orbit determination; multiple solutions; identification

\section{Introduction}

For the last decade, the current surveys have generated a large volume of data, which is increasing with time, and given rise to huge databases of asteroids. However, a large fraction of these asteroids have only been observed over an arc of a few days to a few weeks. When the orbit determination procedure is applied to these data, only low quality orbits can be got. As a consequence, as time goes by and this situation goes on and on for a long time, such asteroids are to be considered lost, that is, they cannot be recovered by pointing the telescope at the predicted position. Therefore, the asteroid databases get polluted with data that belongs to the same physical object, without having an a priori notice of this fact.

This situation requires some action to be taken and the algorithms for asteroid identifications can play an important role. In order to design such algorithms, one needs to take into account the quality, as well as the amount and distribution in time of the available data for the objects to be identified. When there is sufficient observational data to separately solve for two orbits, one for each arc, we will call it orbit identification. In this case the input data are two sets of orbital elements and the identifications are proposed taking into account the similarity of the orbits according to a suitable metric, 
which is not just the size of the difference in the orbital elements (Milani et al. 2000). If the available observational data only allows for the computation of the orbit for one of the two arcs, while the other lacks it, we have what we call the observation attribution problem. That is, we try to attribute the observations of the arc for which it has not been possible to compute a reliable orbit to the other arc which indeed has an orbit. This is done by performing a comparison between the predicted observations, based on the known orbit, and the observed data of the other arc (Milani et al. 2001).

On the other hand, when an asteroid has just been discovered, its orbit is poorly constrained by observations spanning only a short arc. Thus, even if the least squares procedure provides a nominal solution, other orbits are also possible solutions, in the sense that they are compatible with the observations and the corresponding rms of the residuals are not too far from the minimum. All these orbits belong to the so-called confidence region and what we would like is to find an efficient way of sampling it. To this end we use a one-dimensional segment of a curved line called Line Of Variations (LOV). It is possible to define the LOV in different ways, but the general idea is that it is a kind of spine of the confidence region. Here, we use the set of points of convergence of the constrained differential corrections to define it. The idea of "multiple solutions" sampling the LOV was introduced by Milani (1999) and has been used in different contexts. In this case, we are showing its application in asteroid identifications.

In this paper we will try to provide an idea about how we have contributed both to the theoretical understanding and to the solution of the asteroid identification problem. To this end, it is organized as follows. Section 2 is devoted to review the single solution identification methods, which include two main cases: the orbit identification and the observation attribution algorithms, as well as the interaction between the two of them: the recursive procedure, and the results obtained with their application on the Minor Planet Circular batches of data distributed by the Minor Planet Center (MPC). In Section 3 we describe the multiple solutions method and its application to the asteroid identification problem. Finally, in Section 4 we draw some conclusions about the main characteristics of the algorithms presented.

\section{Single solution algorithms}

In general, the asteroid identification problem deals with two separate sets of astrometric observations and can take different forms depending upon the amount and distribution in time of the available observations and upon the time interval between these two sets of data.

\subsection{Orbit identification algorithm}

A first case appears when each of the two sets contains enough information to solve for all the orbital elements by a least squares fit to the observations. Then, we have what we call the orbit identification problem. In such a case, the question is to find the minimum of the target function for a single orbit fitting all the observations.

That is, let us assume that we have two solutions $X_{1}$ and $X_{2}$, with their corresponding normal matrices $C_{1}$ and $C_{2}$, of two separate orbit determination processes. The cost functions can be expanded as

$$
Q_{i}(X)=Q_{i}\left(X_{i}\right)+\frac{1}{m_{i}}\left(X-X_{i}\right)^{T} C_{i}\left(X-X_{i}\right)+\cdots=Q_{i}^{*}+\Delta Q_{i} \quad i=1,2 .
$$


For them to represent the same object, we have to find the minimum of the joint target function $Q(X)$, which is the sum of the two separate target functions

$$
Q=\frac{1}{m}\left(m_{1} Q_{1}+m_{2} Q_{2}\right)=Q^{*}+\frac{\chi^{2}}{m}
$$

where $Q^{*}$ is the value corresponding to the sum (with suitable weighting) of the two separate minima.

Then, if the linear approximation applies, we can approximate each of them by a quadratic form

$$
\chi^{2} \simeq\left(X-X_{1}\right)^{T} C_{1}\left(X-X_{1}\right)+\left(X-X_{2}\right)^{T} C_{2}\left(X-X_{2}\right),
$$

which results in the joint target function being also approximated by a non-homogeneous quadratic form

$$
\chi^{2} \simeq\left(X-X_{0}\right)^{T} C_{0}\left(X-X_{0}\right)+K,
$$

The most important feature of this procedure is that we are not only computing the most likey solution for the joint problem through this expression

$$
X_{0}=C_{0}^{-1}\left(C_{1} X_{1}+C_{2} X_{2}\right),
$$

where $C_{0}=C_{1}+C_{2}$, but that we are also computing the minimum identification penalty which allows us to assess the uncertainty of the identified solution by means of the following formula

$$
K=\Delta X^{T} C \Delta X
$$

where

$$
C=C_{1}-C_{1} C_{0}^{-1} C_{1}
$$

A more detailed description of the how to obtain these formulae can be found in Milani et al. (2001), Section 2.2.

It is clear that we are dealing with a very difficult problem. First, because the number of asteroids is very large and so we cannot effectively apply differential corrections to all the possible pairs, which can be of the order of billions. A second fact generating difficulties is that even a complete orbit, which is a solution of a least squares fit to all the observations of a given arc, is not the only possible orbit and hence the distance, defined by a suitable metric, between the two orbits must be computed using the uncertainty of both orbits.

Thus, with all this in mind, the orbit identification algorithm proposes identifications using a cascade of four filters, the last three being based upon identification metrics that take into account the difference in the orbits weighted with the uncertainty. Figure 1 shows the scheme of such a procedure.

One thing that needs to be kept in mind when trying to identify asteroids observed over short arcs and/or very separated in time is that the problem can become highly nonlinear. In order to lessen this non-linearity several strategies are followed. As a general rule, we use equinoctial elements, instead of Keplerian elements, because in this way we avoid the non-linearity arising from the singularities for null inclination and eccentricity. We also replace the semimajor axis by the mean motion, since the latter is less sensible to non-linear effects. Finally, once one catalog of orbits has been computed with the full Minor Planet Center batches of data, we propagate it to five different epochs. When we start the comparison in the first filter, a simple distance between the two nominal orbits without taking into account their uncertainty, we select those in a catalog whose epoch is the closest to the arithmetic mean of the central times of the observations of each orbit. 


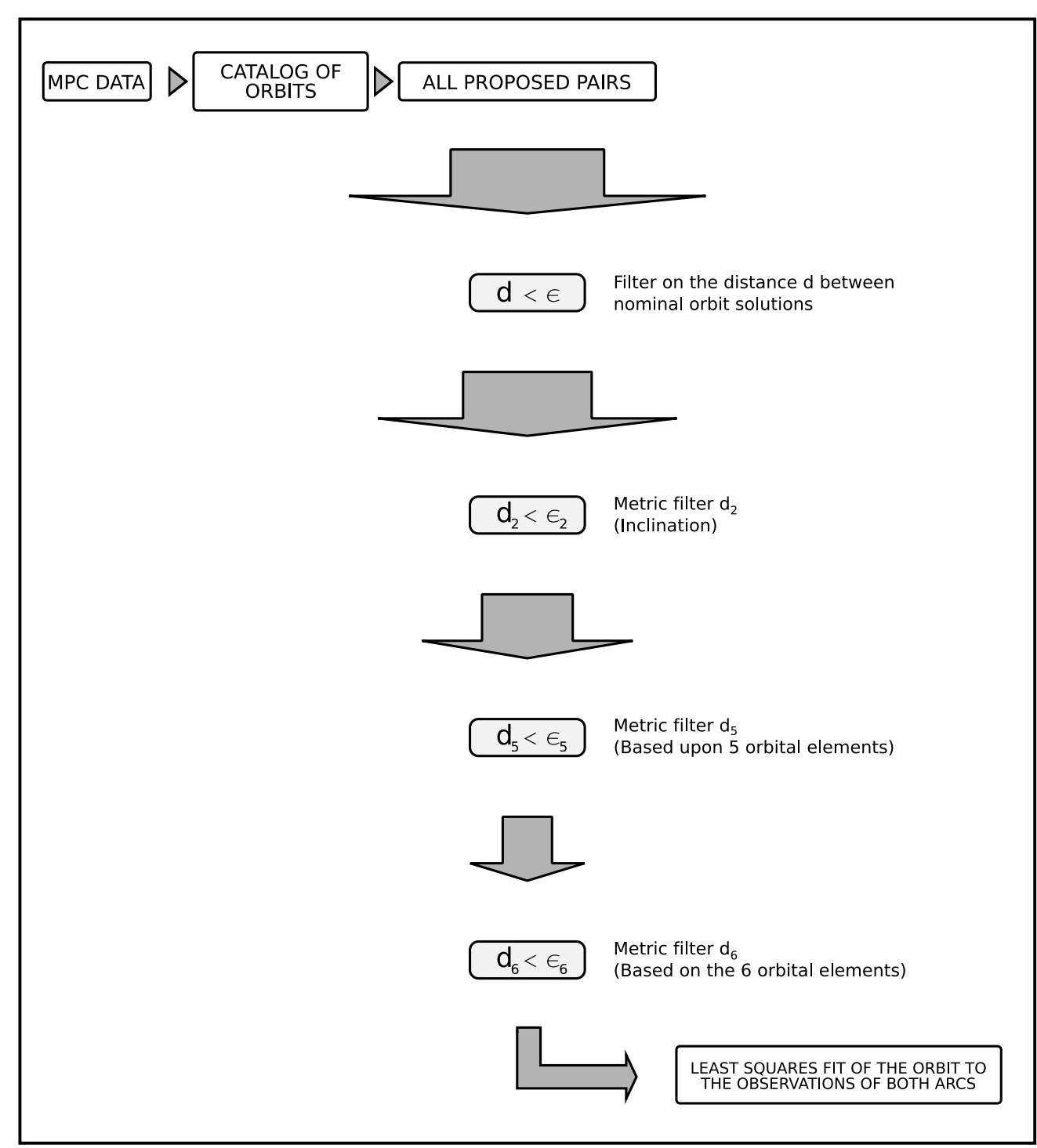

Figure 1. Orbit identification problem - The procedure.

All the pairs passing this first test are sent to the subsequent filter, which essentially looks for similarity in inclination. Later on, a metric based upon five orbital elements is applied and, finally, the last filter that takes into account all the six orbital elements establish the pairs which are sent to the least squares fit of the observations of both arcs.

\subsection{Observation attribution algorithm}

Another case in the asteroid identification problem appears when the amount of data, insufficient to compute an orbit for one arc, is compared to the orbit already computed for the other arc. This is what we call observation attribution problem.

The mathematical formulae for this algorithm are pretty similar to those presented in the previous case, the main difference being that in the orbit identification method we are working in the six-dimensional space of elements, while now we have switched to the four-dimensional space of observations, and so we will not include them again (for 
a complete description of the formulation of this problem, the reader should see Milani et al. 2001).

The underlying idea of the algorithm is easily explained as follows. Each observation has a footprint on the sky (we are assuming it to be a 2 arcsec radius circle). Likewise, any observation prediction starting from an already computed orbit has an uncertainty ellipse: if these two regions overlap, both in the space of positions on the celestial sphere and in that of the apparent motions, we can rate it as a likely identification and submit it to the differential corrections procedure.

The main point here lies in finding a suitable "observation" which can represent all the observations of the second arc. To this end, we define what we call an attributable, which contains information not only on the position of the asteroid on the sky, but also on its apparent motion. More specifically, the attributable contains the time $t_{m}$ that will be referred to as the time of the attributable and is defined as the arihmetic mean of the times of all the observations, the position and proper motion at epoch $t_{m}$ and the position of the asteroid on the sky at a rounded epoch $t_{r}$, which is defined as $t_{r}=k \Delta t$, $k$ being an integer and $\Delta t$ a fixed quantity (10 days as default value).

As in the previous algorithm, we also use a cascade of filters, as described in Figure 2, so that the first only compares the position on the sky at the rounded epoch $t_{r}$, while the second performs the comparison in both the position space and the apparent motion space at the exact epoch $t_{m}$ of the attributable.

\subsection{Recursive algorithm}

Once some identifications have been found with either of the previous two algorithms and the new multi-opposition (or at least improved) orbits are available, we are in a better position to get further identifications for them by applying the observation attribution method again. Figure 3 depicts the logic of this algorithm.

As an example of such a procedure, we are presenting here the best result we have got so far: the identification of seven observation sets

$1998 S P_{61}=2000 C K_{108}=1972 L H=1984 H F_{2}=1986 V Q_{6}=1988 G L_{1}=1990 U Q_{1}$

corresponding to the main-belt asteroid (15427) Shabas.

\subsection{Results}

Table 1 shows a summary of the number of identifications obtained by means of the observation attribution method (first column), the orbit identification algorithm (third column) and the recursive procedure (second and fourth columns). The last column gives the total number of identifications which have been submitted to and published by the MPC.

Table 1. Overall Results

\begin{tabular}{lccccc}
\hline & $\begin{array}{c}\text { Short arc obs. } \\
\text { attributions }\end{array}$ & $\begin{array}{c}\text { Attributions to } \\
\text { the attributions }\end{array}$ & $\begin{array}{c}\text { Orbit } \\
\text { Identifications }\end{array}$ & $\begin{array}{c}\text { Attributions to } \\
\text { the orbit ids. }\end{array}$ & TOTAL \\
\hline Submitted & 4,976 & 433 & 5,070 & 638 & 11,117 \\
Published & 4,509 & 400 & 4,587 & 627 & 10,123 \\
\hline
\end{tabular}

Table 2 provides a detail for the attribution results considering whether the attributed observations where One Night Stands (ONS) or observations obtained during multiple nights. 


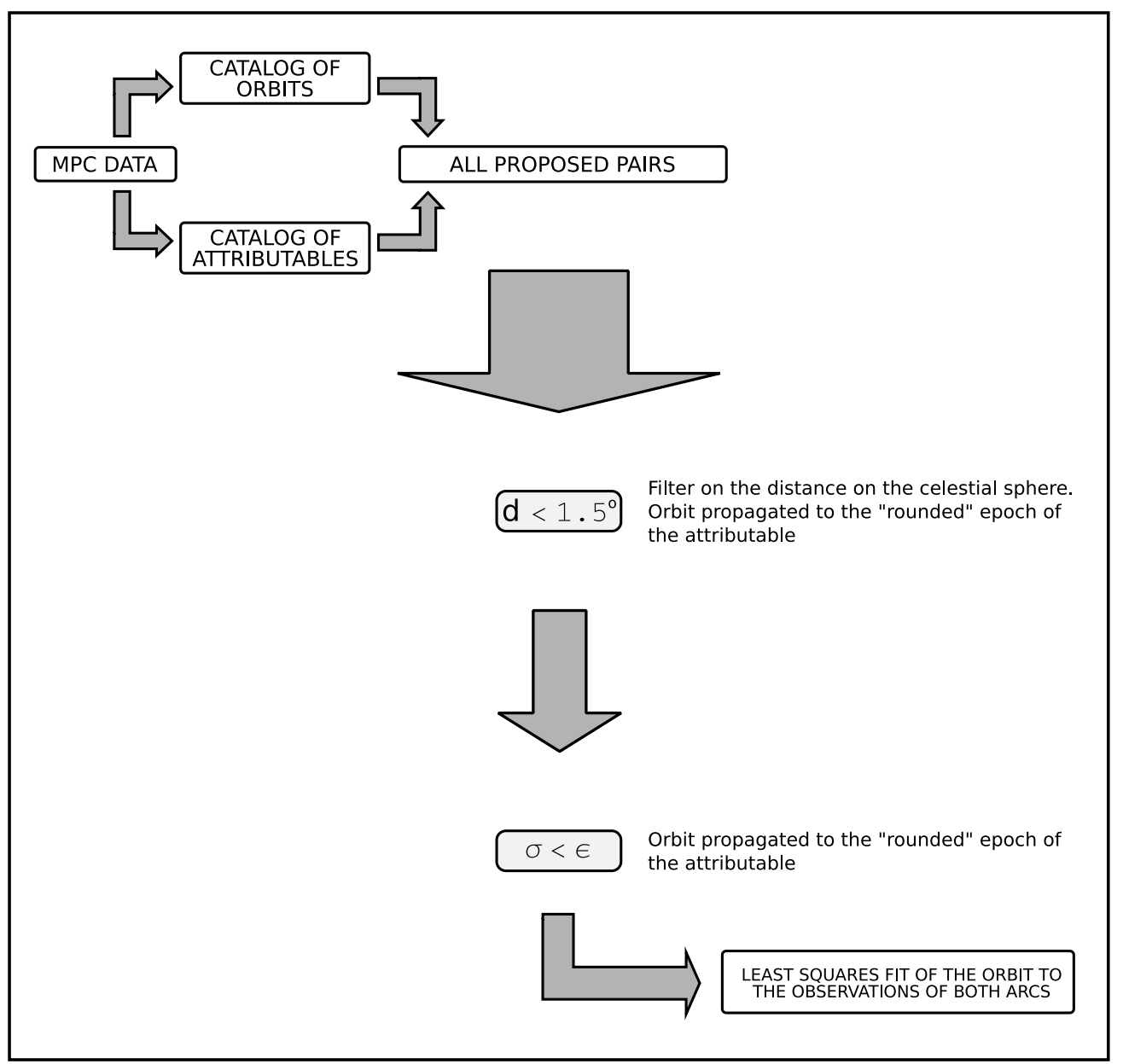

Figure 2. Observation attribution problem - The procedure.

Table 2. Attribution Results

\begin{tabular}{lccc}
\hline & $\begin{array}{c}\text { ONS } \\
\text { attributions }\end{array}$ & $\begin{array}{c}\text { Multiple-Nights } \\
\text { attributions }\end{array}$ & TOTAL \\
\hline Submitted & 2,344 & 3,065 & 5,409 \\
Published & 1,963 & 2,949 & 4,912 \\
Pending & 95 & 26 & 121 \\
\hline
\end{tabular}

All these identifications have been obtained by using as input the Minor Planet Circular batches of data released by the MPC. In order to apply them to the set of One Night Stands, also distributed by the MPC, we first need to assign a uniform designation to the ONS since, as distributed by the MPC, they keep the original name chosen by the observer at the moment of submission.

Once the ONS have been provided with a designation suitable for the OrbFit software, the observation attribution algorithm can be used to look for ONS attributions. When applied to known NEAs we need to take into account their bigger apparent motion so that, in the first filter of the attribution method, we use a smaller $\Delta t$ value (0.001 days). 


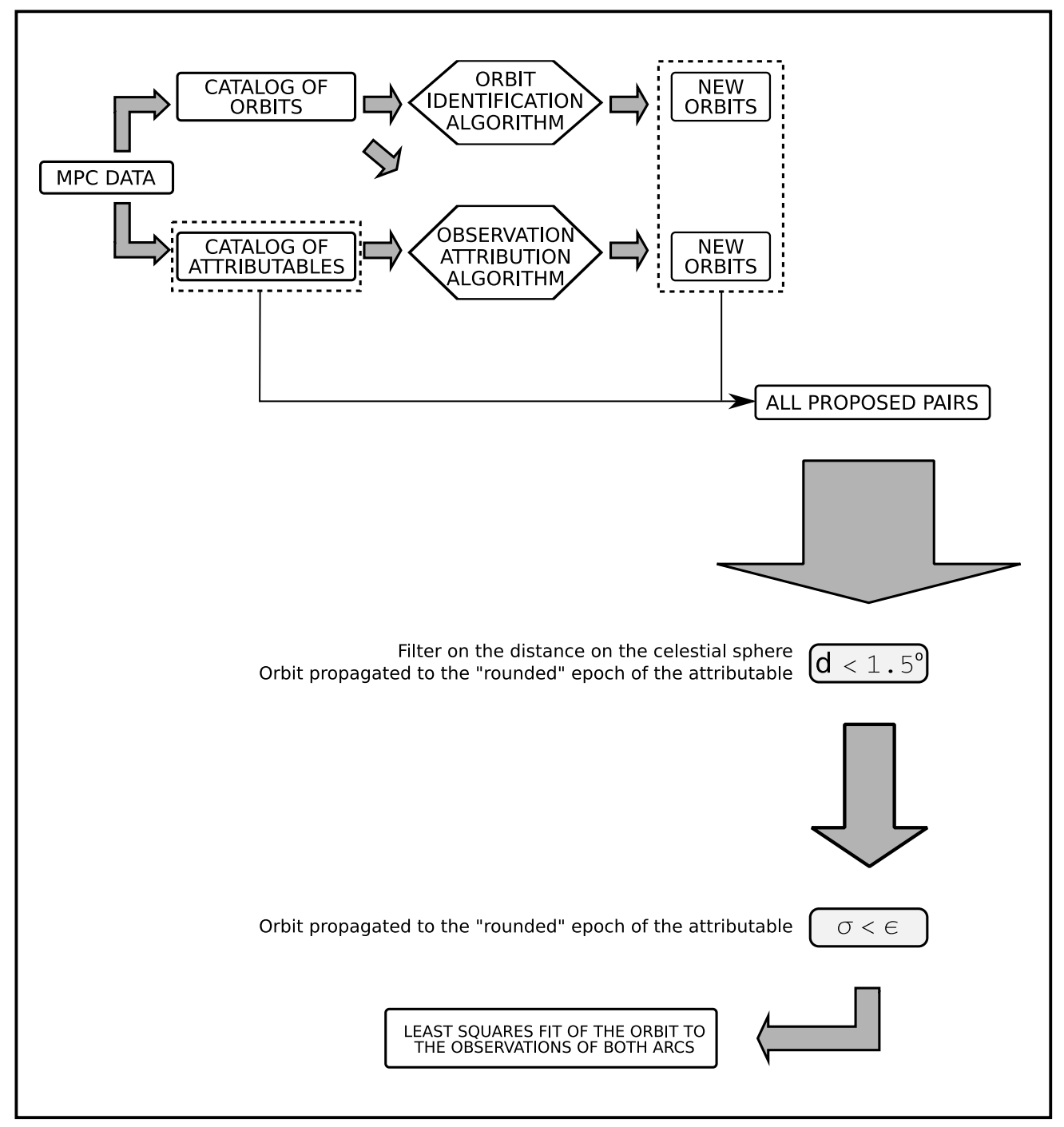

Figure 3. Interaction of algorithms - Recursive procedure.

Proceeding in this way we have obtained a total of about 2,000 ONS attributed both to numbered and unnumbered asteroids.

\section{Multiple solution identification methods}

This Section describes the multiple solutions method, a one dimensional sampling of the orbital confidence region, and its applications in the identification problem. The underlying idea is to record multiple solutions along a one-dimensional subset of the confidence region, known as Line Of Variations (or LOV).

To explain mathematically what the LOV is, let us start by fixing the notation in relation with the well known computation of the nominal solution. The starting point is a fixed data set formed by $m$ measurements of an asteroid. Then, for each orbit we can compute the residuals vector $\Xi=\left(\xi_{1}, \ldots, \xi_{m}\right)$ that measures the deviation between 
measured and computed values. The cost function

$$
Q=\frac{1}{m} \Xi^{T} W \Xi
$$

built using the residuals vector, is a sum of cuadratic terms in which a weighting can be included through the symmetric, positive-definite matrix W.

The least squares fit consists, basically, in finding the stationary points of the cost function. The solution of the corresponding non-linear equations

$$
\frac{\partial Q}{\partial X}=0
$$

are usually found using some iterative procedure. The standard Newton's method involves the computation of the second derivatives of the residuals with respect to the orbital elements, which is computationally expensive. Hence, a variant known as differential correction is generally used. In this method the correction $X \rightarrow X+\Delta X$ is computed as

$$
\Delta X=C^{-1} D
$$

where the normal matrix $C$ and the vector $D$ are defined using the design matrix $B=\frac{\partial \Xi}{\partial X}$ as

$$
C=B^{T} W B \quad \text { and } \quad D=-B^{T} W \Xi .
$$

Whichever procedure is used, if the iterative method converges, the limit $X^{*}$ is a stationary point of the cost function. If, in addition, $X^{*}$ is a local minimum of $Q(X)$, it is called a best-fitting or nominal solution.

\subsection{The confidence region}

Now, let us suppose that for some data set the method of differential corrections has been succesful in providing a nominal orbit. A confidence region $Z(\chi)$ is defined by setting an upper limit to the penalty $\Delta Q$, the increment in the cost function with respect to its value on the nominal solution:

$$
Z(\chi)=\left\{X \mid \Delta Q(X)=Q(X)-Q\left(X^{*}\right) \leqslant \chi^{2} / m\right\} .
$$

When the confidence region and the residuals are small, the cost function can be well approximated using a second order development and under these assumptions the confidence ellipsoid

$$
Z_{L}(\chi)=\left\{X \mid \Delta X^{T} C\left(X^{*}\right) \Delta X \leqslant \chi^{2}\right\}
$$

provides a good estimation of the confidence region. The longest semiaxis of this ellipsoid has the direction of the unit vector $V_{1}$, which is an eigenvector of the normal matrix $C\left(X^{*}\right)$ computed at the nominal solution that corresponds to its smallest eigenvalue $\lambda_{1}$. We say that $V_{1}$ defines the weak direction because in that direction the cost function exhibits the smallest variation and hence represents a weak restriction for the potential solutions of the least squares fit.

\subsection{The Line Of Variations}

The dicussion above could be equally performed when we choose any other point in the confidence region different from the nominal $X^{*}$ and this allows us to define the weak direction vector field as $F=k V_{1}$, where $k=1 / \sqrt{\lambda_{1}}$. Note that the eigenspace associated to $\lambda_{1}$ does not define the unit vector $V_{1}$ uniquely. However, on a simply connected set it is always possible to select a continuous section of a one dimensional distribution. This allows for the practical construction of $F$ by selecting arbitrarily one out of the two possible values of $V_{1}$ at a given point $X$ and extending the vector field by continuity. 
In this context, the LOV is defined as the integral curve of the weak direction vector field with initial condition equal to the nominal solution. Intuitively, the LOV can be thought of as the dorsal spine of the confidence region. If the linear approximation applies, it is the major axis of the confidence ellipsoid. When the linear approximation does not apply, the LOV is indeed curved and can only be computed by numerical integration of the differential equation defining it. The main disadvantage of this definition of the LOV is that it relies heavily on the existence of a nominal solution, which is not guaranteed for all cases and, in addition, there exist a lot of instabilities in its numerical computation which cannot be easily solved by brute force.

So, the question is, how to address the problems associated to the non-existence of a nominal solution. A way out is to consider the algorithm of constrained differential corrections [Milani et al. 2005], which consists in performing differential corrections on the orthogonal hyperplane to the weak direction

$$
H(X)=\left\{Y \mid(Y-X) \cdot V_{1}(X)=0\right\} .
$$

In this case the correction is computed by means of

$$
\Delta X=C^{-1} \pi_{H(X)} D(X),
$$

where $\pi_{H(X)}$ is denoting the orthogonal projection on the hyperplane $H(X)$. The convergence of this method is equivalent to the $D$ vector, defined in the algorithm of ordinary differential corrections (3.1), having the same direction as the vector $V_{1}$ giving the weak direction. This fact suggests to define the LOV as the set of points in which $D$ is parallel to the weak direction:

$$
\mathrm{LOV}=\left\{X|D(X)| \mid V_{1}(X)\right\} .
$$

This new definition matches the previous one when there exists a nominal solution but has the advantage that the non-existence of the latter is no longer an intrinsical obstruction for the construction of the LOV.

So, how do we sample and parameterize the LOV? The condition (3.2), which defines the LOV, effectively imposes five restrictions on the six orbital elements allowing for a one-dimensional solution, that is, a differentiable curve. Unfortunately, there is no direct method to obtain analytically the solution of those equations. In practice, what we do is schematically depicted in Figure 4: we start at a point $X(\sigma)$ (it could be the nominal) and perform an Euler step to get a point $X^{\prime}$, which is not on the LOV (unless it is a straight line). Then we take $X^{\prime}$ as initial condition for the constrained differential corrections that at convergence will provide another point $X^{\prime \prime}$ on the LOV and iterate the process.

Another related problem is the selection of a natural parameterization of the LOV. If $\sigma$ is the value of the parameter corresponding to $X$ then we can use $\sigma+\delta \sigma$ to approximate the parameter value for $X^{\prime \prime}$. Obviously, the situation is easier to handle when there is a nominal solution available. In such a case it is posible to use the quantity $\chi$, associated to the penalty in the cost function, to construct a canonical parameterization for the LOV. In this situation, the probabilistic interpretation of the $\chi$ parameter also provides a simple criteria to decide the moment in which the sampling of the LOV must be terminated. In the linear approximation, both parameters, $\sigma$ and $\chi$, are essentially the same.

\subsection{Results of the tests of identification}

Let us see how the two procedures introduced above, the constrained differential corrections and the multiple solutions method, can be applied in the asteroid identification problem. It is obvious that the more orbits we have computed, the better results can be obtained with the orbit identification method. Thus, what we have done is to adapt the 


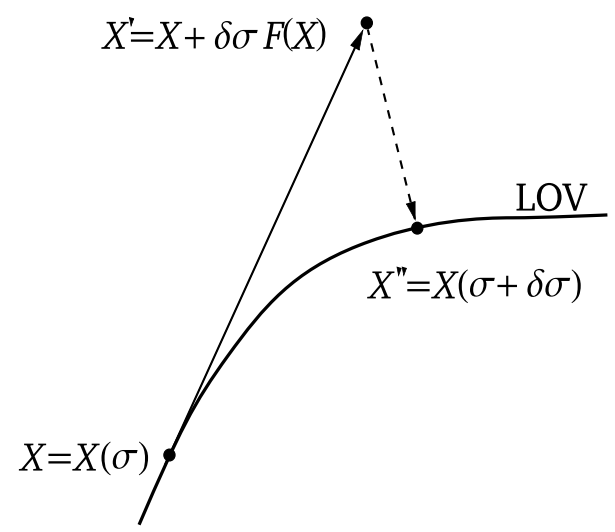

Figure 4. Parameterizing the LOV

orbit identification algorithm described in Subsection 2.1 to also be used with multiple solutions.

In order to quantify the improvement provided by the new techniques we have carried out three full computations using a given data set of unnumbered asteroids. The results of these tests are summarized in Figure 5.

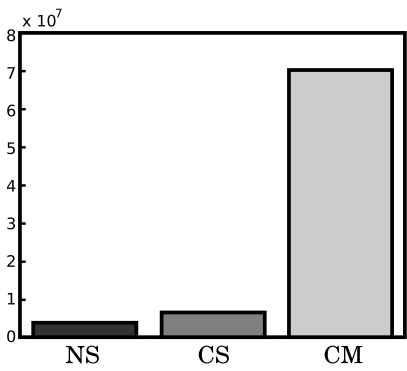

Number of proposed pairs
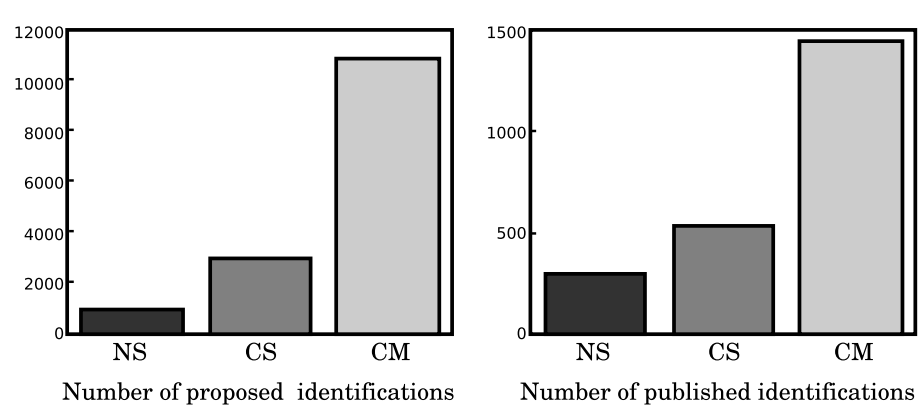

Figure 5. Summary of the tests' results

In each plot, the first bar (labeled NS for Nominal-Single solution), refers to the test that uses the orbit identification method on a catalog of nominal orbits computed with the classical differential correction method. The second bar (labeled CS for ConstrainedSingle solution) represents the results of the test in which the orbit identification algorithm is applied to a catalog of orbits built with the constrained differential corrections method. Finally, the third bar (labeled CM for Constrained-Multiple solutions) gives the numbers for the test in which the multiple solution orbit identification algorithm has been used on the same extended catalog of orbits. For each test, the height of the bars depicts the number of proposed pairs (pairs passing the three filters), number of proposed identifications (i.e., passing differential corrections) and number of published identifications.

All three plots in Figure 5 clearly state the superiority of the method using multiple solutions. Leaving apart its good performance in the number of proposed identifications, the achievements obtained in the published identifications plot are really conclusive: CM multiplies by a factor of three the results of CS and by five those of NS. Therefore, the tests are showing the positive effect of the combination of the two new techniques for the identification task. 
Table 3. Analysis of the identifications proposed by the CS and CM methods.

\begin{tabular}{lcccc}
\hline PROP. IDS. & $\begin{array}{c}\text { Only in } \\
\text { CS }\end{array}$ & $\begin{array}{c}\text { Only in } \\
\text { CM }\end{array}$ & $\begin{array}{c}\text { In } \\
\text { CS \& CM }\end{array}$ & TOTAL \\
\hline & $7.0 \%$ & $73.9 \%$ & $19.1 \%$ & $100 \%$ \\
Not in NS & $6.5 \%$ & $71.9 \%$ & $13.5 \%$ & $91.9 \%$ \\
$1 \mathrm{C}$ & $4.5 \%$ & $31.7 \%$ & $7.9 \%$ & $44.1 \%$ \\
$2 \mathrm{C}$ & $0.8 \%$ & $4.4 \%$ & $1.0 \%$ & $6.2 \%$ \\
\hline
\end{tabular}

Table 3 highlights the role played by the constrained differential corrections. The third and fourth rows show the percentage of identifications in which one or both of the involved asteroids have an orbit computed by means of that procedure. A significant fraction of the identifications obtained with the methods based on constrained solutions involve constrained orbits. As a matter of fact, in more than half of the proposed identifications not detected by the NS method one or both of the orbits were constrained. When we restrict our attention to the published identifications, as shown in Table 4, the corresponding fraction reaches $40 \%$, which is still a substantial contribution.

Table 4. As in Table 3, but for published identifications.

\begin{tabular}{lcccr}
\hline PUB. IDS. & $\begin{array}{c}\text { Only in } \\
\text { CS }\end{array}$ & $\begin{array}{c}\text { Only in } \\
\text { CM }\end{array}$ & $\begin{array}{c}\text { In } \\
\text { CS \& CM }\end{array}$ & TOTAL \\
\hline & $2.7 \%$ & $63.2 \%$ & $34.1 \%$ & $100 \%$ \\
Not in NS & $2.3 \%$ & $60.9 \%$ & $16.6 \%$ & $79.8 \%$ \\
$1 \mathrm{C}$ & $1.5 \%$ & $20.6 \%$ & $7.7 \%$ & $29.8 \%$ \\
$2 \mathrm{C}$ & $0.07 \%$ & $2.0 \%$ & $0.3 \%$ & $2.4 \%$ \\
Credited & $1.3 \%$ & $35.6 \%$ & $13.6 \%$ & $50.5 \%$ \\
\hline
\end{tabular}

A comparative analysis of the behavior of the filters exhibited by the different algorithms shows a sensible variation in the values associated to identifications simultaneously found by more than one method. Generically speaking, there is an overall decreasing in the filter values obtained by the CM procedure with respect to the CS one. This feature becomes more remarkable as the quality of the filters increases, that is, towards the end of the filter procedure. In practical terms, it implies a significant reduction in the optimal cutting values associated to the more selective filters of the CM method. This means that the multiple solutions method notably reduces the identification penalty between orbits, which also implies a reduction in the non-linearity of the problem and finally helps to understand the success of the CM test.

\section{Conclusions}

We have presented several algorithms to deal with the asteroid identification problem discussing their range of applicability and their practical implementation. We have also pointed out the most relevant results obtained using these techniques and we have compared its performance.

The following is a summary of the main conclusions that can be drawn from the results:

- The orbit identification method is more powerful than the observation attribution algorithm, mainly due to the fact that it makes use of more information, since we are 
working in the six-dimensional space of orbital elements, whereas in the observation attribution method we are in the four-dimensional space of the observations.

- The constrained differential correction method greatly improves the orbital determination process, which also means better results when looking for new asteroid identifications.

- The multiple solutions algorithm significantly decreases the non-linearity of the asteroid identification problem and this implies a greater number of successful identifications. It is worth mentioning that the results got with this algorithm are better both from a quantitative and a qualitative point of view, since it not only finds more identifications, but also allows us to obtain some difficult ones which otherwise would be missed.

Despite the advances introduced by the new identification methods, the problem is far from being completely solved. Time performance of the algorithms and reliability of the proposed identification are some of the areas that need to be improved to face the challenge the new large asteroid surveys, promising mammoth quantities of nightly generated data, will pose in the near future.

\section{Acknowledgements}

This research has been funded by the Observatorio de Mallorca (OAM).

\section{References}

Milani, A. 1999, Icarus 137, 269

Milani, A., La Spina, A., Sansaturio, M. E. \& Chesley, S. R. 2000, Icarus 144, 39

Milani, A., Sansaturio, M. E. \& Chesley, S. R. 2001, Icarus 151, 150

Milani, A., Sansaturio, M. E., Tommei, G., Arratia, O. \& Chesley, S. R. 2005, Astron. Astrophys. 431,729 